\title{
Comparison of Eulerian Vlasov Solvers
}

\author{
F. Filbet ${ }^{*} \quad$ E. Sonnendrücker ${ }^{\dagger}$
}

July 31, 2001

\begin{abstract}
Vlasov methods which instead of following the particle trajectories solve the Vlasov equation on a grid of phase space have proven to be an efficient alternative to the Particle In Cell method for some specific problems, in particular those where a good precision is needed for the distribution function in regions of phase space where it is small.

Gridded Vlasov methods have the advantage of being completely free of numerical noise, however the discrete formulations contain some other numerical artifacts, like damping, which are necessary for such methods to be stable and deal with filamentation which is inherent to the Vlasov-Poisson equations. We shall compare in this paper different types of methods solving the Vlasov equation on a grid in phase space: the semi-Lagrangian method, the finite volume method, the spectral method, and a method based on a finite difference scheme conserving exactly several invariants of the system. Moreover for each of those classes of methods, we shall compare different interpolation or reconstruction procedures in this respect, always keeping in mind the cost in memory as well as in CPU time is a very important issue because of the size of the problem which is defined on a grid in phase space which can be up to six-dimensional.
\end{abstract}

PACS codes: $52.25 . \mathrm{Dg}, 52.65 .-\mathrm{y}$.

Keywords: Vlasov - Eulerian methods - numerical simulation - comparison.

A model which can be used in many cases for the study of plasma as well as of beam propagation is the Vlasov equation coupled with the Maxwell equations or some reduced model to compute the self consistent fields. It describes the evolution of a system of particles under the effects of external and self-consistent fields. The unknown $f(t, x, v)$, depending on the time $t$, the position $x$, and the velocity $v$, represents the distribution of particles in phase space for each species. The numerical resolution of the Vlasov equation is usually performed by particle methods (PIC) which consist in approximating the plasma by a finite number of particles. Trajectories of these particles are computed from characteristic curves given by the Vlasov equation, whereas self-consistent fields are

\footnotetext{
*IECN, INRIA, Université Henri Poincaré, Nancy

${ }^{\dagger}$ IRMA, Université Louis Pasteur, Strasbourg
} 
computed on a mesh of the physical space. This method allows to obtain satisfying results with a relatively small number of particles. However, it is well known that the numerical noise inherent to the particle method becomes, in some cases, too important to have an accurate description of the distribution function in phase space. Moreover, the numerical noise only decreases in $1 / \sqrt{N}$, when the number of particles $N$ is increased.

To remedy this problem, methods discretizing the Vlasov equation on a mesh of phase space have been proposed. Among them, the semi-Lagrangian method consists in computing the distribution function at each grid point by following the characteristic curves ending at this grid point backward in time for one time step and then interpolate the distribution function at the obtained point from the neighboring grid points values, knowing that the distribution function is conserved along characteristic curves. To compute the origin of the characteristic a high order interpolation method is needed. A special case of this method, based on a time splitting enabling an exact computation of the characteristics and a cubic spline interpolation, has been first introduced by Cheng and Knorr [4], and subsequently used in many plasma physics papers, see for example [7, 10] and references therein. This method has then been cast into the more general framework of semi-Lagrangian methods by E. Sonnendrücker et al. [14]. This method was also successfully applied to beam physics problems, namely the simulation of space charge waves on an initially semi-Gaussian beam and halo development in a uniform focusing channel [15]. Another flavor of the semi-Lagrangian method was introduced by Nakamura and Yabe and called the Cubic Interpolated Propagation (CIP) method. It is based on a Hermite interpolation for which the gradients of the distribution function are also advanced along the characteristics [16]. This method needs the storage of $f, \nabla_{x} f$, and $\nabla_{v} f$, therefore in order not to be to much memory consuming the mesh on which it is applied should be coarser.

Another type of scheme for the Vlasov equation is the finite volume type method (or flux balance method), where the discrete unknowns consist of averages of the distribution function on volumes paving the phase space, and it is updated by considering fluxes entering and leaving each volume and is thus perfectly conservative. The first scheme of this type was introduced by Boris and Book [2], and another implementation more recently by [8]. We recently proposed an improved version of this scheme that we called the Positive and Flux Conservative method (PFC [9], which is designed, in addition to be conservative, to preserve the positivity and the maximum value of the distribution function. The scheme was implemented up to third order accuracy.

We shall also consider the Fourier-Fourier spectral method introduced by Klimas and Farell $[5,6]$, and based on a splitting between physical space and velocity space transport, using at each step a backward and forward Fourier transport for the shift. A filtration algorithm to limit small scale filamentation was also proposed in this scheme.

One of the flaws common to all Vlasov solvers is their smearing of small structures and the associated non physical increase of entropy. However, this feature is necessary for the stability of Eulerian Vlasov solvers. Indeed, a finite difference scheme based on a method introduced by Arakawa [1] which conserves $\int f^{2} d x d v$ numerically becomes unstable when filamentation phenomena occur. The method can still be useful when we stabilize it by a collision term which is chosen so as to still conserve desired moments of the distribution function.

In this paper, we recall the methods introduced above, and propose in particular for the semiLagrangian scheme new interpolation techniques in particular local ones which should be more 
efficient for parallel computation as they need less communication between processors. Moreover, in order to get some insight on the behavior of these methods we compare them for some classical problems of plasma physics, with respect to numerical dissipation, phase errors and accuracy at lower resolution as well computational cost for one specific problem.

\section{The Vlasov equation.}

The evolution of the density of particles $f(t, x, v) d x d v$ in the phase space $(x, v) \in \mathbb{R}^{d} \times \mathbb{R}^{d}$, $d=1, . ., 3$, is given by the Vlasov equation,

$$
\frac{\partial f}{\partial t}+v \cdot \nabla_{x} f+F(t, x, v) \cdot \nabla_{v} f=0
$$

which can also be written in the following conservative form

$$
\frac{\partial f}{\partial t}+\operatorname{div}_{x}(v f)+\operatorname{div}_{v}(F(t, x, v) f)=0
$$

The force field $F(t, x, v)$ can consist of an applied external field and a self consistent field which is coupled with the distribution function $f$ giving a nonlinear system. Typically $F=\frac{q}{m}\left(E_{s e l f}+\right.$ $E_{\text {appl }}+v \times\left(B_{\text {self }}+B_{\text {appl }}\right)$, where the self fields are solution of Maxwell's equations with sources induced by the particles, or in simplified cases which we shall consider in this article, the magnetic field is neglected and the self electric field is computed using the Poisson equation, i.e.

$$
E_{\text {self }}(t, x)=-\nabla_{x} \phi(t, x), \quad-\varepsilon_{0} \Delta_{x} \phi=\rho,
$$

where $m$ represents the mass of one particle, $q$ its charge and $\rho$ is defined by

$$
\rho(t, x)=q \int_{\mathbb{R}^{d}} f(t, x, v) d v .
$$

\section{The Flux Conservative method.}

The starting point of our algorithm is the Flux Balance method [8], discretizing the Vlasov equation in the conservative form: we first observe that using a time splitting scheme the algorithm boils down to one dimensional problems which have the following form,

$$
\partial_{t} f+\partial_{x}(\mathbf{u}(t, x) f)=0, \quad \forall(t, x) \in \mathbb{R}^{+} \times\left[x_{\min }, x_{\max }\right] .
$$

We will assume that $\mathbf{u}(t, x)$ is smooth enough. Then we can define the characteristic curves solution of the differential system corresponding to the transport equation:

$$
\left\{\begin{array}{l}
\frac{d \mathrm{x}}{d s}(s)=\mathbf{u}(s, \mathrm{x}(s)), \\
\mathrm{x}(t)=x .
\end{array}\right.
$$


Let us denote by $\mathrm{x}(s, t, x)$ the solution of (6) and define the Jacobian $J(s, t, x)=\partial_{x} \mathrm{x}(s, t, x)$. In [3], it is proved that $J(s, t, x)$ is positive for all $(s, t, x) \in \mathbb{R}^{+} \times \mathbb{R}^{+} \times \mathbb{R}$, and the solution of the transport equation (5) reads

$$
f(t, x)=f(s, \mathrm{x}(s, t, x)) J(s, t, x),
$$

which describes the conservation of particles along the characteristic curves

$$
\int_{K} f(t, x) d x=\int_{\mathbf{x}(s, t, K)} f(s, x) d x
$$

for any interval $K$, where

$$
\mathrm{x}(s, t, K)=\{y \in \mathbb{R}: \quad y=\mathrm{x}(s, t, z) ; \quad z \in K\} .
$$

Note that this property remains true for dimensions $d \geq 1$. Now, let us introduce a finite set of mesh points $\left(x_{i+1 / 2}\right)_{i \in I}$ of the computational domain $\left(x_{\min }, x_{m a x}\right)$, we will denote the space step by $\Delta x=x_{i+1 / 2}-x_{i-1 / 2}$, and $C_{i}=\left[x_{i-1 / 2}, x_{i+1 / 2}\right]$. Assume the values of the distribution function are known at time $t^{n}=n \Delta t$. We find the new values at time $t^{n+1}$ by integrating of the distribution function on each cell. Indeed using the conservation of particles (7) on each interval $C_{i}$ from time $t^{n}$ to time $t^{n+1}$, we have

$$
\int_{x_{i-1 / 2}}^{x_{i+1 / 2}} f\left(t^{n+1}, x\right) d x=\int_{\mathrm{x}\left(t^{n}, t^{n+1}, x_{i-1 / 2}\right)}^{\mathrm{x}\left(t^{n}, t^{n+1}, x_{i+1 / 2}\right)} f\left(t^{n}, x\right) d x .
$$

Denoting by

$$
\Phi_{i+1 / 2}\left(t^{n}\right)=\int_{\mathrm{x}\left(t^{n}, t^{n+1}, x_{i+1 / 2}\right)}^{x_{i+1 / 2}} f\left(t^{n}, x\right) d x
$$

The flux conservation becomes

$$
\int_{x_{i-1 / 2}}^{x_{i+1 / 2}} f\left(t^{n+1}, x\right) d x=\Phi_{i-1 / 2}\left(t^{n}\right)+\int_{x_{i-1 / 2}}^{x_{i+1 / 2}} f\left(t^{n}, x\right) d x-\Phi_{i+1 / 2}\left(t^{n}\right) .
$$

The evaluation of the average of the solution over $\left[x_{i-1 / 2}, x_{i+1 / 2}\right]$ smears out neatly fine details of the exact solution which cannot be computed anyway on a finite size grid for longer times for which sufficient mesh refinement is way to costly.

In order to get an accurate high order scheme, an essential step is now to choose an efficient method to reconstruct the distribution function from the values on each cell $C_{i}$.

\subsection{The Flux Balance Method (FBM).}

In [8], E. Fijalkow only used a linear interpolation

$$
f_{h}(x)=f_{i}+\left(x-x_{i}\right) \frac{f_{i+1}-f_{i-1}}{2 \Delta x}, \quad \forall x \in\left(x_{i-1 / 2}, x_{i+1 / 2}\right) .
$$

This method is very straightforward to implement. However, its drawbacks are that it does not give a positive approximation and does not control spurious oscillations. 


\subsection{The Positive and Flux Conservative method (PFC).}

This method was introduced recently in [9]. It is based on a reconstruction via primitive function: let $F\left(t^{n}, x\right)$ be a primitive of the distribution function $f\left(t^{n}, x\right)$, we will denote by

$$
f_{i}^{n}=\frac{1}{\Delta x} \int_{x_{i-1 / 2}}^{x_{i+1 / 2}} f\left(t^{n}, x\right) d x
$$

then we have $F\left(t^{n}, x_{i+1 / 2}\right)-F\left(t^{n}, x_{i-1 / 2}\right)=\Delta x f_{i}^{n}$, and

$$
F\left(t^{n}, x_{i+1 / 2}\right)=\Delta x \sum_{k=0}^{i} f_{k}^{n}=w_{i}^{n} .
$$

On the interval $\left[x_{i-1 / 2}, x_{i+1 / 2}\right]$, we use the stencil $\left\{x_{i-3 / 2}, x_{i-1 / 2}, x_{i+1 / 2}, x_{i+3 / 2}\right\}$ to approximate the primitive by a polynomial of degree three. By differentiation, we define a first approximation $\tilde{f}_{h}\left(t^{n}, x\right)$, which is a third order approximation of the distribution function $f\left(t^{n}, x\right)$. Note that $\tilde{f}_{h}\left(t^{n}, x\right)$ is a perfectly valid third order approximation of $f$. However, it does not verify the property of the exact solution of the Vlasov equation that $0 \leq f(t, x) \leq f_{\infty}$ for all $x$ and $t$, where $f_{\infty}$ is the maximum value of the initial distribution function $f_{0}$. In order to enforce this property, we introduce slope correctors to obtain for all $x \in C_{i}$,

$$
\begin{aligned}
f_{h}\left(t^{n}, x\right)=f_{i}^{n} & +\frac{\epsilon_{i}^{+}}{6 \Delta x^{2}}\left[2\left(x-x_{i}\right)\left(x-x_{i-3 / 2}\right)+\left(x-x_{i-1 / 2}\right)\left(x-x_{i+1 / 2}\right)\right]\left(f_{i+1}^{n}-f_{i}^{n}\right) \\
& +\frac{\epsilon_{i}^{-}}{6 \Delta x^{2}}\left[2\left(x-x_{i}\right)\left(x-x_{i+3 / 2}\right)+\left(x-x_{i-1 / 2}\right)\left(x-x_{i+1 / 2}\right)\right]\left(f_{i}^{n}-f_{i-1}^{n}\right),
\end{aligned}
$$

with

$$
\epsilon_{i}^{+}= \begin{cases}\min \left(1 ; 2 f_{i}^{n} /\left(f_{i+1}^{n}-f_{i}^{n}\right)\right) & \text { if } f_{i+1}^{n}-f_{i}^{n}>0 \\ \min \left(1 ;-2\left(f_{\infty}-f_{i}^{n}\right) /\left(f_{i+1}^{n}-f_{i}^{n}\right)\right) & \text { if } f_{i+1}^{n}-f_{i}^{n}<0\end{cases}
$$

and

$$
\epsilon_{i}^{-}= \begin{cases}\min \left(1 ; 2\left(f_{\infty}-f_{i}^{n}\right) /\left(f_{i}^{n}-f_{i-1}^{n}\right)\right) & \text { if } f_{i}^{n}-f_{i-1}^{n}>0, \\ \min \left(1 ;-2 f_{i}^{n} /\left(f_{i}^{n}-f_{i-1}^{n}\right)\right) & \text { if } f_{i}^{n}-f_{i-1}^{n}<0 .\end{cases}
$$

It is easy to verify that the approximation of the distribution function $f_{h}\left(t^{n}, x\right)$ previously constructed satisfies

- Conservation of the average: for all $i \in I, \quad \int_{x_{i-1 / 2}}^{x_{i+1 / 2}} f_{h}\left(t^{n}, x\right) d x=\Delta x f_{i}^{n}$.

- Maximum principle: for all $x \in\left(x_{\min }, x_{\max }\right), \quad 0 \leq f_{h}\left(t^{n}, x\right) \leq f_{\infty}$.

$>$ From this reconstruction, we approximate the quantity $\Phi_{i+1 / 2}\left(t^{n}\right)$, by looking for the cell $C_{j}$ such that $\mathrm{x}\left(t^{n}, t^{n+1}, x_{i+1 / 2}\right) \in C_{j}$ and setting $\alpha_{i}=x_{j+1 / 2}-\mathrm{x}\left(t^{n}, t^{n+1}, x_{i+1 / 2}\right)$. Then for a positive $u(t, x)$, 
we obtain

$$
\begin{aligned}
& \Phi_{i+1 / 2}\left(t^{n}\right)=\int_{x_{j+1 / 2}-\alpha_{i}}^{x_{i+1 / 2}} f\left(t^{n}, x\right) d x=\Delta x \sum_{k=j+1}^{i} f_{k}^{n}+ \\
& +\alpha_{i}\left[f_{j}^{n}+\frac{\epsilon_{j}^{+}}{6}\left(1-\frac{\alpha_{i}}{\Delta x}\right)\left(2-\frac{\alpha_{i}}{\Delta x}\right)\left(f_{j+1}^{n}-f_{j}^{n}\right)+\frac{\epsilon_{j}^{-}}{6}\left(1-\frac{\alpha_{i}}{\Delta x}\right)\left(1+\frac{\alpha_{i}}{\Delta x}\right)\left(f_{j}^{n}-f_{j-1}^{n}\right)\right],
\end{aligned}
$$

and when $\mathbf{u}(t, x)$ is negative, we set $\alpha_{i}=x_{j-1 / 2}-\mathrm{x}\left(t^{n}, t^{n+1}, x_{i+1 / 2}\right)$, then $-\Delta x \leq \alpha_{i} \leq 0$ and

$$
\begin{aligned}
& \Phi_{i+1 / 2}\left(t^{n}\right)=\int_{x_{j-1 / 2}-\alpha_{i}}^{x_{i+1 / 2}} f\left(t^{n}, x\right) d x=\Delta x \sum_{k=i+1}^{j-1} f_{k}^{n}+ \\
& +\alpha_{i}\left[f_{j}^{n}-\frac{\epsilon_{j}^{+}}{6}\left(1-\frac{\alpha_{i}}{\Delta x}\right)\left(1+\frac{\alpha_{i}}{\Delta x}\right)\left(f_{j+1}^{n}-f_{j}^{n}\right)-\frac{\epsilon_{j}^{-}}{6}\left(2+\frac{\alpha_{i}}{\Delta x}\right)\left(1+\frac{\alpha_{i}}{\Delta x}\right)\left(f_{j}^{n}-f_{j-1}^{n}\right)\right] .
\end{aligned}
$$

\section{The semi-Lagrangian method (SL).}

This method is based on the usual advective form of the Vlasov equation, which reads

$$
\frac{\partial f}{\partial t}+v \cdot \nabla_{x} f+F(t, x) \cdot \nabla_{v} f=0, \quad \forall(t, x, v) \in \mathbb{R}^{+} \times \mathbb{R}^{d} \times \mathbb{R}^{d},
$$

where $F$ is the force field. As the distribution function solution of the Vlasov equation is constant along the particle trajectories, assuming it is known at time $t^{n}=n \Delta t$, the solution at time $t^{n+1}$ is given by

$$
f\left(t^{n+1}, x, v\right)=f\left(t^{n}, X\left(t^{n}, t^{n+1}, x, v\right), V\left(t^{n}, t^{n+1}, x, v\right)\right),
$$

where $\left(X\left(t^{n}, t^{n+1}, x, v\right), V\left(t^{n}, t^{n+1}, x, v\right)\right)$ stands for the solution of the differential system defining the characteristic curves (which are the particle trajectories) and which reads

$$
\begin{aligned}
& \frac{d X}{d t}=V(t), \\
& \frac{d V}{d t}=F(X(t), t) .
\end{aligned}
$$

The semi-Lagrangian method consists in approximating the distribution function at each grid point of the computational domain $\left(\mathbf{x}_{i}, \mathbf{v}_{i}\right)_{i \in I}$, and updating it at each time step with its value at the origin of the characteristic $\left(X\left(t^{n}, t^{n+1}, \mathbf{x}_{i}, \mathbf{v}_{i}\right), V\left(t^{n}, t^{n+1}, \mathbf{x}_{i}, \mathbf{v}_{i}\right)\right)$, which is computed from values on the grid using a high order interpolation method. In previous works $[4,14]$, a cubic spline interpolation has been used. This gives very good results, but has the drawback of being non local which causes a higher communication overhead on parallel computers. We want here to compare its properties with those of local interpolation procedures.

Actually, the semi-Lagrangian method for the Vlasov equation can be simplified a lot when a splitting procedure is used, because in this case the feet of the characteristics can be computed 
explicitly at each split step. The different split steps for advancing from time step $t^{n}$ to $t^{n+1}$ are the following:

$$
\begin{aligned}
f^{*}(x, v) & =f\left(t^{n}, x-v \Delta t / 2, v\right), \\
f^{* *}(x, v) & =f^{*}\left(x, v-E^{*}(x) \Delta t\right), \\
f\left(t^{n+1}, x, v\right) & =f^{* *}(x-v \Delta t / 2, v),
\end{aligned}
$$

where $E^{*}(x)$ is computed from $f^{*}$.

For simplicity, we will only consider one dimensional reconstruction, but it can be easily generalized to higher dimensions. In the following discussion, we will assume the distribution function is known at time $t^{n}$ on the grid:

$$
f\left(t^{n}, \mathbf{x}_{i}\right)=f_{i}^{n}, \quad \forall i \in I,
$$

and will present two methods of reconstruction based on the Lagrange and Hermite interpolation.

\subsection{The Lagrange interpolation method.}

We look for a continuous approximation $f$ of $f\left(t^{n},.\right)$ such that

$$
\forall i \in I, f\left(\mathbf{x}_{i}\right)=f_{i}^{n}, \text { and } \forall x \in\left[\mathbf{x}_{i}, \mathbf{x}_{i+1}\right], \quad f(x)=q_{m}(x),
$$

where $q_{m}(x)$ belongs to $P_{2 m+1}\left[\mathbf{x}_{i}, \mathbf{x}_{i+1}\right]$ i.e. the set of polynomial of degree $2 m+1$ on the interval $\left[\mathbf{x}_{i}, \mathbf{x}_{i+1}\right]$. We only choose polynomials of odd degree to have a centered approximation, indeed the set of points used to construct the polynomial $q_{m}(x)$ on the interval $\left[\mathbf{x}_{i}, \mathbf{x}_{i+1}\right]$ is

$$
\left\{\mathbf{x}_{i-m}, . ., \mathbf{x}_{i}, \mathbf{x}_{i+1}, \ldots, \mathbf{x}_{i+1+m}\right\}
$$

and $q_{m}(x)$ is in the following form

$$
q_{m}(x)=f_{i-m}^{n}+\sum_{k=1}^{2 m+1} f\left[\mathbf{x}_{i-m}, \ldots, \mathbf{x}_{i-m+k}\right] \prod_{l=0}^{k}\left(x-\mathbf{x}_{i-m+l}\right),
$$

where $f\left[\mathbf{x}_{i-m}, \ldots, \mathbf{x}_{i-m+k}\right]$ is given by the divided difference formula

$$
\begin{aligned}
& f\left[\mathbf{x}_{i}, \ldots, \mathbf{x}_{i+p}\right]=\frac{1}{p !} \frac{f\left[\mathbf{x}_{i+1}, . ., \mathbf{x}_{i+p}\right]-f\left[\mathbf{x}_{i}, . ., \mathbf{x}_{i+p-1}\right]}{\mathbf{x}_{i+p}-\mathbf{x}_{i}} \\
& f\left[\mathbf{x}_{i}\right]=f\left(\mathbf{x}_{i}\right) .
\end{aligned}
$$

This interpolation method only gives a continuous function, then we have to consider a high degree polynomial ( $m$ greater than 2 ) to obtain an accurate approximation of the distribution function. It has been implemented until $m=4$.

$>$ From this reconstruction, we define the approximation of the distribution function $f\left(t^{n+1}, \mathbf{x}_{i}\right)$ at time $t^{n+1}$ at each grid point as follows: 
for simplicity take $m=2$, assume $X\left(t^{n}, t^{n+1}, \mathbf{x}_{i}\right)$ is known and belongs to the interval $\left[\mathbf{x}_{j}, \mathbf{x}_{j+1}\right]$, then let us denote by $\alpha_{i}=\left[X\left(t^{n}, t^{n+1}, \mathbf{x}_{i}\right)-\mathbf{x}_{j}\right] / \Delta x$, with $\Delta x=\mathbf{x}_{j+1}-\mathbf{x}_{j}$,

$$
\begin{aligned}
f\left(t^{n+1}, \mathbf{x}_{i}\right)= & q_{2}\left(X\left(t^{n}, t^{n+1}, \mathbf{x}_{i}\right)\right) \\
= & f_{j}^{n}+\alpha_{i}\left[f_{j+1}^{n}-f_{j}^{n}\right]-\frac{1}{2} \alpha_{i}\left(1-\alpha_{i}\right)\left[f_{j+1}^{n}-2 f_{j}^{n}+f_{j-1}^{n}\right] \\
& -\frac{1}{6} \alpha_{i}\left(1-\alpha_{i}\right)\left(1+\alpha_{i}\right)\left[f_{j+2}^{n}-3 f_{j+1}^{n}+3 f_{j}^{n}-f_{j-1}^{n}\right] \\
& +\frac{1}{24} \alpha_{i}\left(1-\alpha_{i}\right)\left(1+\alpha_{i}\right)\left(2-\alpha_{i}\right)\left[f_{j+2}^{n}-3 f_{j+1}^{n}+3 f_{j}^{n}-f_{j-1}^{n}\right] \\
& +\frac{1}{120} \alpha_{i}\left(1-\alpha_{i}\right)\left(1+\alpha_{i}\right)\left(2-\alpha_{i}\right)\left(2+\alpha_{i}\right)\left[f_{j+2}^{n}-4 f_{j+1}^{n}+6 f_{j}^{n}-4 f_{j-1}^{n}+f_{j-2}^{n}\right] .
\end{aligned}
$$

In the general situation, the semi-Lagrangian method does not conserve global mass, but for linear advection with constant coefficients, the use of a centered approximation allows to ensure the conservation of global mass: for simplicity, assume the propagation velocity $u$ is positive,

$$
f\left(t^{n+1}, \mathbf{x}_{i}\right)=f\left(t^{n}, \mathbf{x}_{i}-u \Delta t\right)
$$

we set $j=\left[\frac{u \Delta t}{\Delta x}\right]$, where [.] represents the integer part, and $0 \leq \alpha=u \Delta t-\mathbf{x}_{i-j} \leq \Delta x$, then for the previous scheme, we have

$$
\sum_{i} f_{i}^{n+1}=\sum_{i} f_{i-j}^{n}+\alpha \sum_{i}\left[f_{i-j+1}^{n}-f_{i-j}^{n}\right]+\frac{1}{2} \alpha(1-\alpha) \sum_{i}\left[f_{i-j+1}^{n}-2 f_{i-j}^{n}+f_{i-j-1}^{n}\right]+\ldots
$$

using the divided difference formula, we obtain the result

$$
\sum_{i} f_{i}^{n+1}=\sum_{i} f_{i-j}^{n}=\sum_{i} f_{i}^{n} .
$$

\subsection{The Hermite interpolation method.}

In this section, we will only consider cubic polynomials to construct a $C^{1}$ approximation $f\left(t^{n},.\right)$ using a Hermite interpolation, which needs to estimate the derivative $\partial_{x} f(x)$. In [16], authors treated the case when the propagating velocity is constant and proposed to approximate the profile of the derivative by differentiating the equation. They finally obtained a transport equation for $f$, and $\partial_{x} f$, but the memory cost is increased to treat the Vlasov equation in the $(x, v)$ space. Here, we propose to approximate the derivative by a fourth order accurate finite difference formula:

$$
\partial_{x} f_{i}^{n}=\frac{1}{12 \Delta x}\left[8\left[f_{i+1}^{n}-f_{i-1}^{n}\right]-\left[f_{i+2}^{n}-f_{i-2}^{n}\right]\right] .
$$

Then, for all $x \in\left[\mathbf{x}_{i}, \mathbf{x}_{i+1}\right], f(x)$ is given by the cubic polynomial $p_{3}(x)$ such that

$$
\begin{array}{ll}
p_{3}\left(\mathbf{x}_{i}\right)=f_{i}^{n}, & \partial_{x} p_{3}\left(\mathbf{x}_{i}\right)=\partial_{x} f_{i}^{n} \\
p_{3}\left(\mathbf{x}_{i+1}\right)=f_{i+1}^{n}, & \partial_{x} p_{3}\left(\mathbf{x}_{i+1}\right)=\partial_{x} f_{i+1}^{n}
\end{array}
$$


Then, setting $\alpha_{i}=\left[X\left(t^{n}, t^{n+1}, \mathbf{x}_{i}\right)-\mathbf{x}_{j}\right] / \Delta x$, where $X\left(t^{n}, t^{n+1}, \mathbf{x}_{i}\right)$ belongs to $\left[\mathbf{x}_{j}, \mathbf{x}_{j+1}\right]$, the value at time $t^{n+1}$ is determined by

$$
\begin{aligned}
f\left(t^{n+1}, \mathbf{x}_{i}\right)= & p_{3}\left(X\left(t^{n}, t^{n+1}, \mathbf{x}_{i}\right)\right) \\
= & f_{i}^{n}+\alpha_{i}\left[f_{i+1}^{n}-f_{i}^{n}\right]+\alpha_{i}^{2}\left[3\left[f_{i+1}^{n}-f_{i}^{n}\right]-\Delta x\left[2 \partial_{x} f_{i}^{n}-\partial_{x} f_{i+1}^{n}\right]\right] \\
& +\alpha_{i}^{3}\left[\Delta x\left[\partial_{x} f_{i+1}^{n}+\partial_{x} f_{i}^{n}\right]-2\left[f_{i+1}^{n}-f_{i}^{n}\right]\right] .
\end{aligned}
$$

Let us note that using the centered approximation of the derivative, we also prove the conservation of global mass for the linear advection with constant coefficients.

\subsection{The CIP method.}

This method, which is a variant of the semi-Lagrangian method, was developed by Nakamura and Yabe [16]. It is based on a splitting procedure into one-dimensional advection equations. The interpolation step in the semi-Lagrangian algorithm is perform using a cubic Hermite interpolation, i.e. an interpolation using on each interval the values of the functions and its derivatives at the endpoints of the interval. Its specificity is that the derivatives needed for such an interpolation procedure are not computed numerically but advanced themselves along the one dimensional characteristics. For example, the first split step of the Vlasov-Poisson approximation consists in solving the following system,

$$
\begin{array}{r}
\frac{\partial f}{\partial t}+v \frac{\partial f}{\partial x}=0 \\
\frac{\partial\left(\partial_{x} f\right)}{\partial t}+v \frac{\partial\left(\partial_{x} f\right)}{\partial x}=0 \\
\frac{\partial\left(\partial_{v} f\right)}{\partial t}+\frac{\partial\left(v \partial_{v} f\right)}{\partial x}=0
\end{array}
$$

where the unknowns are $\left(f, \partial_{x} f, \partial_{v} f\right)$.

Steps (14) and (15) just consist in a shift which is exact for the part being advanced, but step (16) requires a numerical procedure which needs to be performed with great care so as not to ruin the accuracy and conservation properties of the algorithm [16]. Once $f$ and its derivatives are advanced the reconstruction step of the semi-Lagrangian algorithm can be performed using a Hermite interpolation.

This scheme has the advantage of being local, which is a nice feature for parallel computations, as it involves less inter-processor communications, however it has a higher memory cost as it needs storing all the derivatives as well as the values of distribution function at each grid point.

\section{A spectral method.}

This method has been proposed by Klimas and Farell to approximate the one dimensional VlasovPoisson and Vlasov-Maxwell system [5, 6]. 
The distribution function is approximated by a partial sum of a Fourier series

$$
f_{N}(t, x, v)=\sum_{k=-N}^{N} \hat{f}_{k}(t, v) \exp (-i 2 \pi k \cdot x / L), \quad \forall x \in(0, L) ;
$$

and the Fourier coefficients are given by

$$
\hat{f}_{k}(t, v)=\sum_{k=-N}^{N} f\left(t, x_{j}, v\right) \exp \left(i 2 \pi k \cdot x_{j} / L\right) ; \quad \forall k \in\{-N, \ldots, N\} .
$$

Then, the first shift of (12) is equivalent to the application of a phase shift to the expansion coefficients

$$
\hat{f}_{k}^{*}(v)=\hat{f}_{k}\left(t^{n}, v\right) \exp (-i 2 \pi k \cdot v \Delta t / 2 L) .
$$

In short, each split step consists in performing a forward FFT, a phase shift, and a backward FFT.

The development of filamentation in the velocity distribution, with its related propagation to large Fourier modes in $v$ in the Fourier-Fourier transformed distribution, can lead to serious computational difficulty in the transformed space. To remedy to this problem, a filtered solution can be computed. It consists in eliminating high frequencies of the velocity distribution, which gives a smoother approximation $[5,6]$.

\section{A Finite Difference Method (FDM).}

In 1966 Arakawa [1] introduced a finite difference method for the integration of the Euler equation for two-dimensional fluid flow which can, in particular, achieve conservation of mean kinetic energy and mean square vorticity. This scheme can be easily adapted to the Vlasov-Poisson equations. In $1 \mathrm{D}$, it reads in a dimensionless form

$$
\frac{\partial f}{\partial t}+v \frac{\partial f}{\partial x}+\frac{\partial \varphi}{\partial x} \frac{\partial f}{\partial v}=0 \quad \Delta \varphi=\int_{\mathbb{R}} f d v-1 .
$$

Setting

$$
\psi=\varphi-\frac{v^{2}}{2} \quad \text { and } \quad J(\psi, f)=\frac{\partial \psi}{\partial x} \frac{\partial f}{\partial v}-\frac{\partial \psi}{\partial v} \frac{\partial f}{\partial x}
$$

the Vlasov equation reads

$$
\frac{\partial f}{\partial t}+J(\psi, f)=0
$$

which is the form used by Arakawa who gives a second and fourth order discretization of $J(\psi, f)$, with the following conservation properties:

- Particle conservation:

$$
\int_{\mathbb{R}^{2}} J_{h}(\psi, f) d x d v=0 \Longrightarrow \int_{\mathbb{R}^{2}} f(t) d x d v=\int_{\mathbb{R}^{2}} f_{0} d x d v
$$


- Energy conservation:

$$
\int_{\mathbb{R}^{2}} J_{h}(\psi, f) \psi d x d v=0 \Longrightarrow \int_{\mathbb{R}^{2}} f(t) \psi(t) d x d v=\int_{\mathbb{R}^{2}} f_{0} \psi(0) d x d v .
$$

- Conservation of the mean square of $f$ :

$$
\int_{\mathbb{R}^{2}} J_{h}(\psi, f) f d x d v=0 \Longrightarrow \int_{\mathbb{R}^{2}} f^{2}(t) d x d v=\int_{\mathbb{R}^{2}} f_{0}^{2} d x d v
$$

Then, we first compute three approximations of $J(\psi, f)$ on the grid $\left(x_{i}, y_{j}\right)_{i, j}$. Denoting by $h=$ $x_{i+1}-x_{i}=y_{j+1}-y_{j}$, we obtain

$$
\begin{aligned}
J_{i, j}^{1}(\psi, f)= & \frac{1}{4 h^{2}}\left[\left(\psi_{i+1, j}-\psi_{i-1, j}\right)\left(f_{i, j+1}-f_{i, j-1}\right)\right. \\
& \left.-\left(\psi_{i, j+1}-\psi_{i, j-1}\right)\left(f_{i+1, j}-f_{i-1, j}\right)\right] \\
J_{i, j}^{2}(\psi, f)= & \frac{1}{4 h^{2}}\left[\psi_{i+1, j}\left(f_{i+1, j+1}-f_{i+1, j-1}\right)-\psi_{i-1, j}\left(f_{i-1, j+1}-f_{i-1, j-1}\right)\right. \\
& \left.-\psi_{i, j+1}\left(f_{i+1, j+1}-f_{i-1, j+1}\right)+\psi_{i, j-1}\left(f_{i+1, j-1}-f_{i-1, j-1}\right)\right] \\
J_{i, j}^{3}(\psi, f)= & \frac{1}{4 h^{2}}\left[\psi_{i+1, j+1}\left(f_{i, j+1}-f_{i+1, j}\right)-\psi_{i-1, j-1}\left(f_{i-1, j}-f_{i, j-1}\right)\right. \\
& \left.-\psi_{i-1, j+1}\left(f_{i, j+1}-f_{i-1, j}\right)+\psi_{i+1, j-1}\left(f_{i+1, j}-f_{i, j-1}\right)\right] .
\end{aligned}
$$

Finally, the approximation $J_{h}(\psi, f)$ is given by computing the average of the above three approximations, allowing to conserve the total mass, the total energy and the $L^{2}$ norm of $f$. However, it does not preserve positivity. Moreover the scheme becomes oscillatory when filaments develop on the order of the cell size. The previously introduced Vlasov solvers, rely on the interpolation procedures to numerically smear out the thin filament and thus damp the oscillations. We can here achieve the same effect by adding to the Vlasov equation a small collision term. Following Robert and Someria [12], we can compute this term so that it maximizes local entropy and conserves moments up to any desired order. In the actual code, we have implemented the conservation of moments up to the second order, i.e. mass, impulsion and kinetic energy. More precisely, we look for a collision model of the form

$$
\frac{\partial f}{\partial t}=\frac{\partial \mathcal{J}}{\partial v}
$$

where $\mathcal{J}$ should be chosen such that at each point in $x$ : collisions maximize entropy

$$
S(t, x)=\int_{\mathbb{R}} f \log f d v,
$$


conserve total number of particles, impulsion, kinetic energy:

$$
\int_{\mathbb{R}} \frac{\partial \mathcal{J}}{\partial v}\left(\begin{array}{c}
1 \\
v \\
v^{2} \\
\cdot \\
\cdot \\
v^{K}
\end{array}\right) d v=0
$$

Moreover, for a given norm

$$
\|\mathcal{J}\|=\int_{\mathbb{R}} \frac{\mathcal{J}^{2}}{f} d v
$$

we require $\|\mathcal{J}\|=\alpha$, where the parameter $\alpha$ is linked to the collision frequency which is here of order to the grid size. Using a Lagrange multiplier technique, these constraints yield

$$
\mathcal{J}=\alpha\left(\frac{\partial f}{\partial v}+\sum_{k=1}^{K} A_{k} k f v^{k-1}\right), \text { with } A_{k}=(m-1) \frac{\int_{\mathbb{R}} f v^{m-2} d v}{\int_{\mathbb{R}} f v^{m+k-2} d v} .
$$

In particular for $K=2$, our model reads

$$
\frac{\partial f}{\partial t}+J(\psi, f)=\alpha \frac{\partial}{\partial v}\left(\frac{\partial f}{\partial v}+A_{1} f-A_{2} f v\right),
$$

with $A_{1}=\frac{u_{0}}{\epsilon-u_{0}^{2} / n}$, and $A_{2}=\frac{n}{\epsilon-u_{0}^{2} / n}$, where

$$
n=\int_{\mathbb{R}} f d v, \quad u_{0}=\frac{1}{n} \int_{\mathbb{R}} f v d v, \quad \epsilon=\int_{\mathbb{R}} f v^{2} d v .
$$

\section{Numerical tests.}

\subsection{The linear advection.}

We first consider the linear advection problem with constant coefficients to analyze the dissipation for the different schemes using a Fourier transform.

Let us first consider the amplification factor for the different methods (see Fig. 1). We observe that methods using a smooth reconstruction (Hermite or spline) are less dissipative than those using only a continuous interpolation. To obtain a similar amplification factor with the Lagrange interpolation as with the spline interpolation, a polynomial of degree nine is required. The dissipation of the conservative method with a quadratic polynomial is identical to the one using cubic Lagrange interpolation. The linear reconstruction used in the (FBM) is the most dissipative. Consider now the phase errors (see Fig. 2). It is the most important for the semi-Lagrangian method using a Hermite reconstruction with a second or fourth order approximation of the derivative. The spline reconstruction is also less accurate than the Lagrange interpolation of degree nine. 


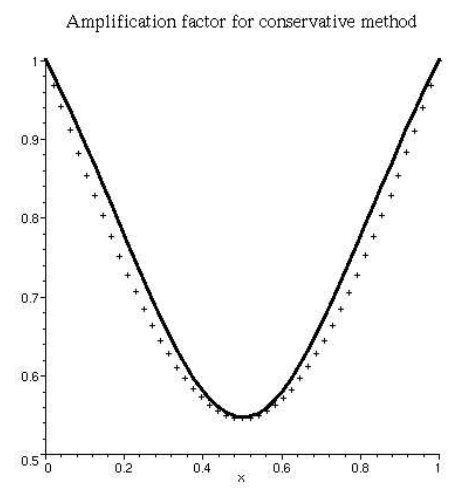

(1)

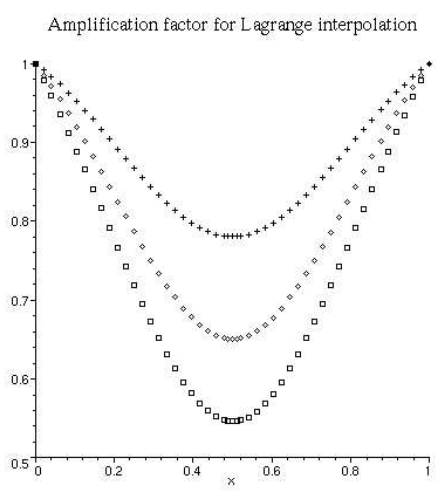

$(2)$

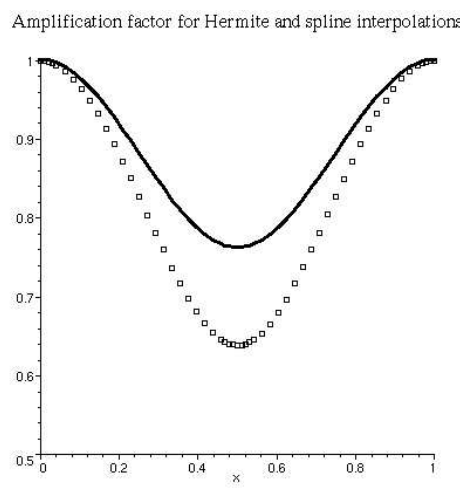

(3)

Figure 1: The amplification factor with respect to $\alpha$ for a fixed mode $k$. (1) the FBM (cross) and third order reconstruction without slope corrector (line); (2) the semi-Lagrangian method with a Lagrange interpolation of degree 3 (box), 5 (diamond), and 9 (cross); (3) and with cubic Hermite polynomial with a fourth order approximation of the derivative (box), and cubic spline interpolation (line).

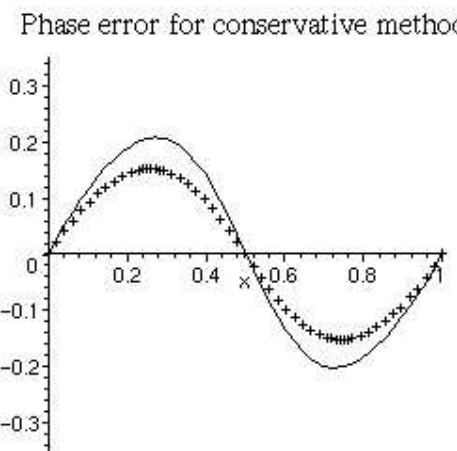

(1)

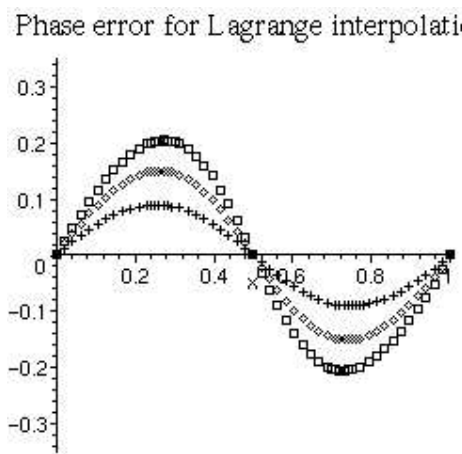

$(2)$

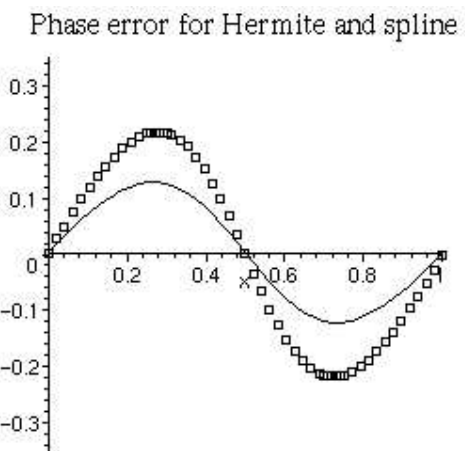

$(3)$

Figure 2: The phase error with respect to $\alpha$ for a fixed mode $k$. (1) the conservative method for the FBM (cross) and third order reconstruction without slope corrector (line); (2) the semi-Lagrangian method with a Lagrange interpolation of degree 3 (box), 5 (diamond), and 9 (cross); (3) and with cubic Hermite polynomial with a fourth order approximation of the derivative (box), and cubic spline interpolation (line). 


\subsection{The one dimensional Vlasov-Poisson system.}

\subsubsection{The plasma echo.}

Following the work by Manfredi et al. [11], we consider an initial data representing an homogeneous Maxwellian distribution

$$
f(0, x, v)=\frac{1}{\sqrt{2 \pi}} \exp \left(-v^{2} / 2\right), \quad \forall(x, v) \in(0, L) \times \mathbb{R},
$$

with $L=2 \pi / k$ and $k=0.483$. At time $t=0$, we excite an external electric field in the plasma, of the form

$$
E_{0}(x)=\alpha \cos (k x)
$$

where $\alpha=0.1$. This field induces a velocity modulation, and right after a density modulation, which eventually decays by Landau damping. After the first has damped away, we launch a second wave at time $t=30 \omega_{p}^{-1}$ of the form

$$
E_{1}(x)=\alpha \cos (2 k x) .
$$

The density modulation induced by this second pulse also fades away. However, after a time much longer than the inverse Landau damping rate of the first two pulses, a third wave appears (the echo) as a modulation of the density at the wave number $k_{e c h o}=2 k-k=k$. The echo is due to the nonlinear interaction between the two pulses and is essentially a phenomenon of beating between two waves. Fig. 3 shows the electrostatic energy as a function of time. The damping of the two pulses and the subsequent echo are accurately reproduced with the different schemes. The echo wave number is indeed $k_{\text {echo }}=k$ as predicted by the theory. The Landau damping rate for the first pulse is in good agreement with the theoretical value $\gamma_{L}=0.4 \omega_{p}^{-1}$ and even larger for the second pulse. The echo time is

$$
t_{\text {echo }}=\frac{2 k}{2 k-k} 30 \omega_{p}^{-1}=60 \omega_{p}^{-1},
$$

which corresponds very well with the numerical value. From time $t=30 \omega_{p}^{-1}$ to $t \simeq 60 \omega_{p}^{-1}$, the second wave has no effect on the first mode of the electric field, but at time $t=60 \omega_{p}^{-1}$, it is strongly perturbed by the echo effect.

We report the results of a simulation using a number of cells $N_{x}=32$ in the $x$-direction, and $N_{v}=64,128$ in the $v$-direction with $v_{\max }=6.5$, and $\Delta t=1 / 8$ for conservative, spectral and semi-Lagrangian methods which are not restricted by a CFL condition, whereas $\Delta t=1 / 40$ for the Finite Difference Method. The numerical solution remains positive for all schemes and the relative error norms of variations of kinetic entropy, $L^{2}$-norm, and total energy always stay less than $10^{-3}$ for semi-Lagrangian and conservative schemes. The evolution of the electric field (until $t=30 \omega_{p}^{-1}$ ) obtained by different schemes is compared with the approximation computed on a fine grid $(512 \times 1024$ points) in Table 1 . The conservative methods seem to be more accurate on a coarse grid, whereas the semi-Lagrangian method with a cubic spline interpolation gives the best result with $32 \times 128$ points. The order of convergence for the different methods agrees very well with the order of the reconstruction. Finally, let us mention that the approximation obtained by Finite Difference Method and the Spectral algorithm respectively strongly depend on the numerical 


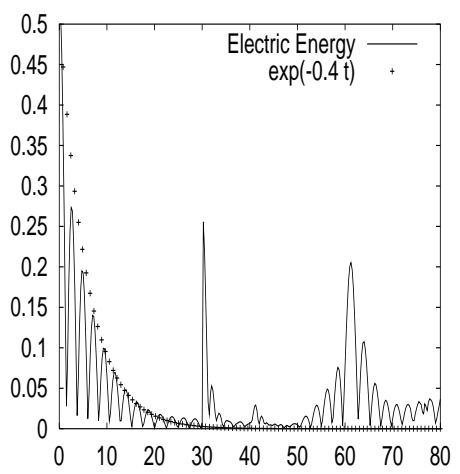

(a)

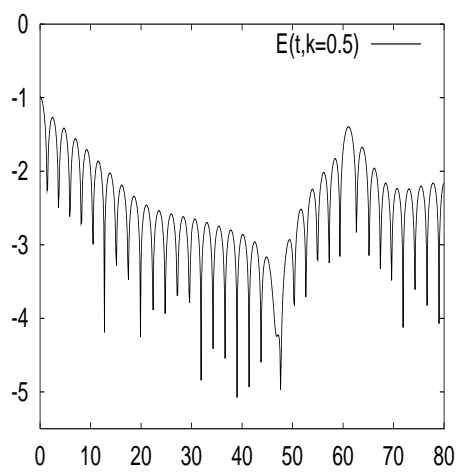

(b)

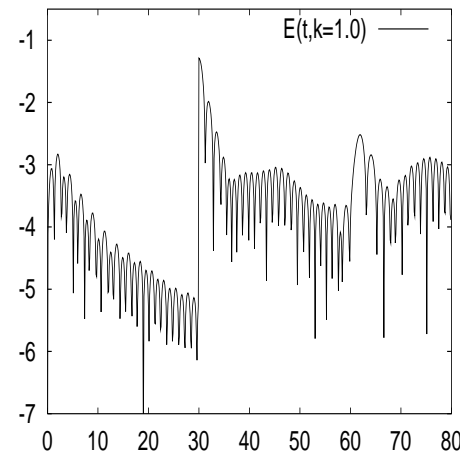

(c)

Figure 3: Time development of (a) the electric energy (b) the first mode of the electric field (in log scale) (c) the second mode of the electric field (in log scale) for the plasma echo test.

\begin{tabular}{|c|c|c|c|c|c|c|c|}
\hline & FBM & PFC & FDM & Spectral & CIP & SL Spline & SL Hermite \\
\hline $32 \times 32$ & 0.05 & 0.045 & 0.09 & 0.056 & 0.071 & 0.078 & 0.070 \\
\hline $32 \times 128$ & 0.0065 & 0.0036 & 0.035 & 0.0035 & 0.0035 & 0.0014 & 0.0034 \\
\hline
\end{tabular}

Table 1: Relative error norm for different methods for $32 \times 64$ and $32 \times 128$ points for the plasma echo test.

collision frequency and on the truncature of low frequencies. It is an inconvenient for the robustness of the methods.

\subsubsection{The nonlinear Landau damping.}

In this case, the initial data is given by

$$
f(0, x, v)=\frac{1}{\sqrt{2 \pi}}(1+\alpha \cos (k x)) \exp \left(-v^{2} / 2\right),
$$

with $\alpha=0.5$, and $L=2 \pi / k$. We are using a number of cells $N_{x}=32$ in the $x$-direction, and $N_{v}=64$ in the $v$-direction with $v_{\max }=6.5$, and $\Delta t=1 / 8$ for conservative and semi-Lagrangian methods which are not restricted by a CFL condition, whereas $\Delta t=1 / 40$ for the Finite Difference Method.

The linear Landau damping theory is valid as long as $t<\alpha^{-1 / 2}$; for longer times the problem is inherently nonlinear. Here, the Landau theory cannot be applied because nonlinear effects are too important, but this test has been studied numerically by many authors $[5,16,11]$. The electric energy first decays exponentially and is next periodically oscillating. In Fig. 4, the electrical energy, obtained by the different methods using $32 \times 64$ points, is plotted in logarithmic scales. It can be compared with an accurate approximation $(512 \times 1024)$ for which $L^{p}$ norms and kinetic entropy are well conserved. The evolution obtained by the PFC scheme clearly appears like the 


\begin{tabular}{|c|c|c|c|}
\hline Numerical method & $32 \times 32$ points & $32 \times 64$ points & $32 \times 128$ points \\
\hline FBM & 03.33 sec. & 05.39 sec. & 10.80 sec. \\
\hline PFC & 03.56 sec. & 06.28 sec. & 11.20 sec. \\
\hline FDM & 17.22 sec. & 35.27 sec. & 71.20 sec. \\
\hline SPECTRAL & 04.10 sec. & 08.25 sec. & 16.90 sec. \\
\hline CIP & 13.83 sec. & 21.40 sec. & 43.24 sec. \\
\hline SL SPLINE & 06.12 sec. & 10.55 sec. & 20.90 sec. \\
\hline SL HERMITE & 03.60 sec. & 06.90 sec. & 11.00 sec. \\
\hline
\end{tabular}

Table 2: Total time of computation for different methods with respect to the number of points points for the nonlinear Landau damping test.

best approximation. Nonlinear effects are so important that it is necessary to control spurious oscillations. The evolution of $L^{p}$ norms of $f_{h}(t), \sum\left|f_{i}(t)\right|^{p}$ for $p=1,2$, are reported in Fig. 5. The PFC scheme conserves the total mass and also positivity, the $L^{1}$ norm of $f_{h}(t)$ is then conserved along time, whereas strong spurious oscillations occur for the different semi-Lagrangian methods and for the spectral method, which do not have as efficient a mechanism to eliminate numerical instabilities and rely on sampling effects for that. The use of slope correctors in the PFC scheme enhances the decay of the discrete $L^{2}$ norm, but when oscillations, due to the nonlinearity, are damped or averaged by the projection on the grid, the $L^{2}$ norm is well stabilized.

For the distribution function in the $(x, v)$ space, small bumps appear around the phase velocity $v_{\phi}=\omega / k$. These bumps represent particles which are trapped by electrostatic waves (see Fig.6). As a consequence of the entropy decay, the distribution function is smoothed when filaments become smaller than the phase space grid size. Nevertheless, this smooth approximation seems to give a good description of macroscopic values (physics quantities obtained by the integration of moments of the distribution function with respect to $v$ ) since the evolution of the electric energy is more accurate than one obtained from the semi-Lagrangian method using the cubic spline interpolation.

Computational cost of the different methods implemented. For the test case of the nonlinear Landau Damping, which is characteristic in this respect, we give for each method the total time of computation, in Table 2 with respect to the number of points. We notice that the numerical schemes using a local reconstruction are faster than ones using a global interpolation. The FDM is penalized by a CFL condition on the time step, which increases the computation time on finer grids. The use of the Fast Fourier Transforms for the spectral algorithm induces an amount of work of $N \log (N)$, where $N$ is the number of unknowns, whereas the computational cost of Flux Conservative and semi-Lagrangian methods is linear with respect to the number of unknowns.

\subsubsection{The two stream instability.}

We consider the symmetric two stream instability with initial condition

$$
f(0, x, v)=\frac{2}{7 \sqrt{2 \pi}}\left(1+5 v^{2}\right)(1+\alpha((\cos (2 k x)+\cos (3 k x)) / 1.2+\cos (k x))) \exp \left(-v^{2} / 2\right),
$$




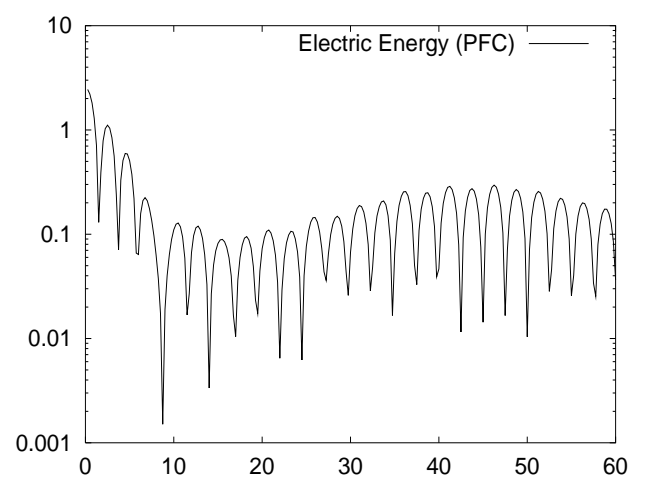

(a)

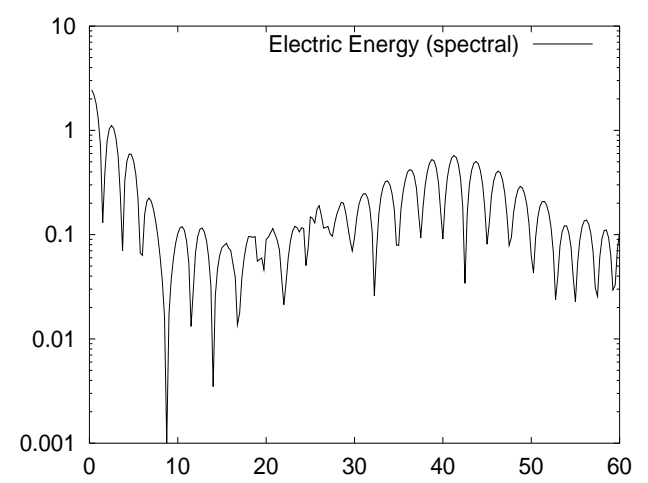

(c)

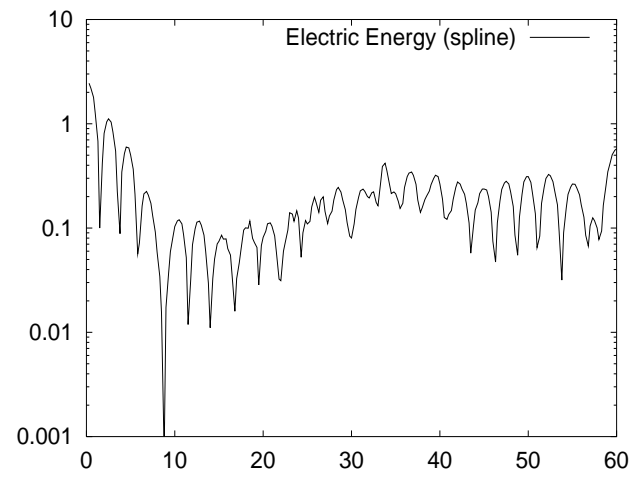

(e)

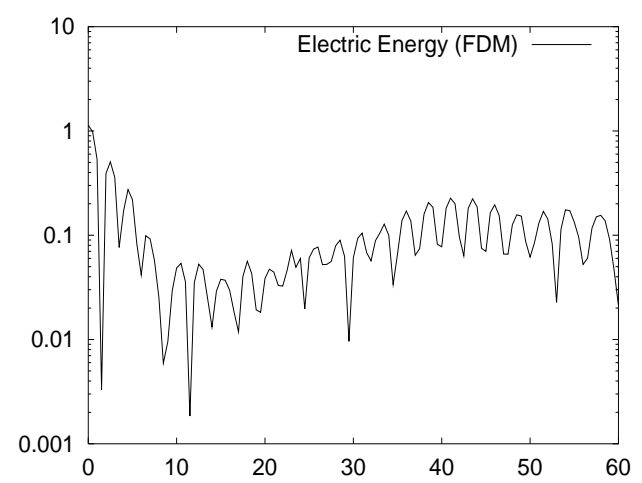

(b)

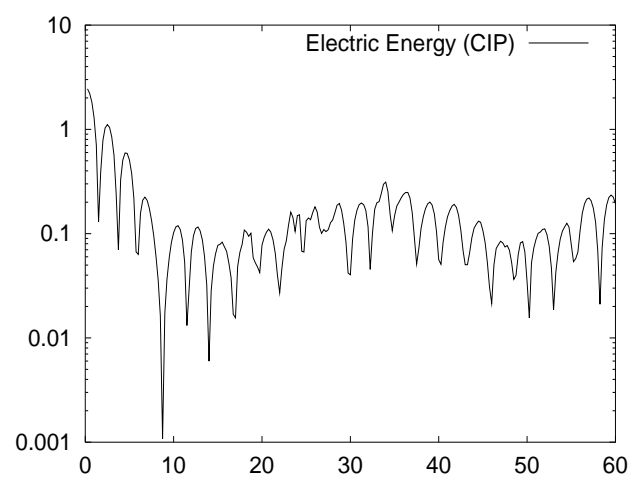

(d)

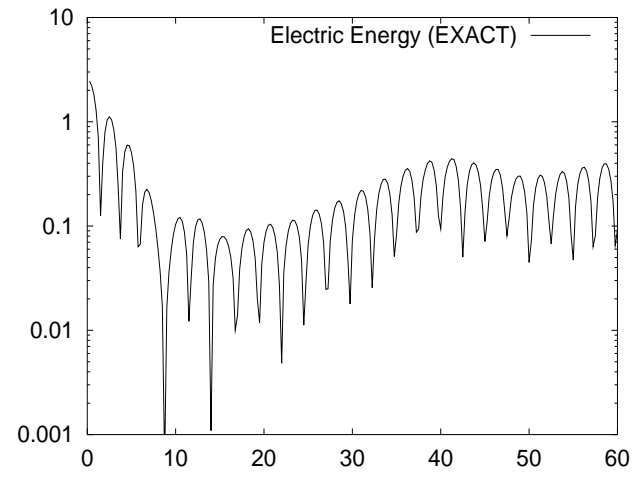

(f)

Figure 4: Time development of the electric energy using $32 \times 64$ points obtained by (a) the PFC scheme, (b) the FDM, (c) the spectral algorithm, (d) the CIP method, (e) the semi-Lagrangian method with a Cubic spline interpolation, $(f)$ the exact solution $(512 \times 1024)$ for the nonlinear Landau damping. 

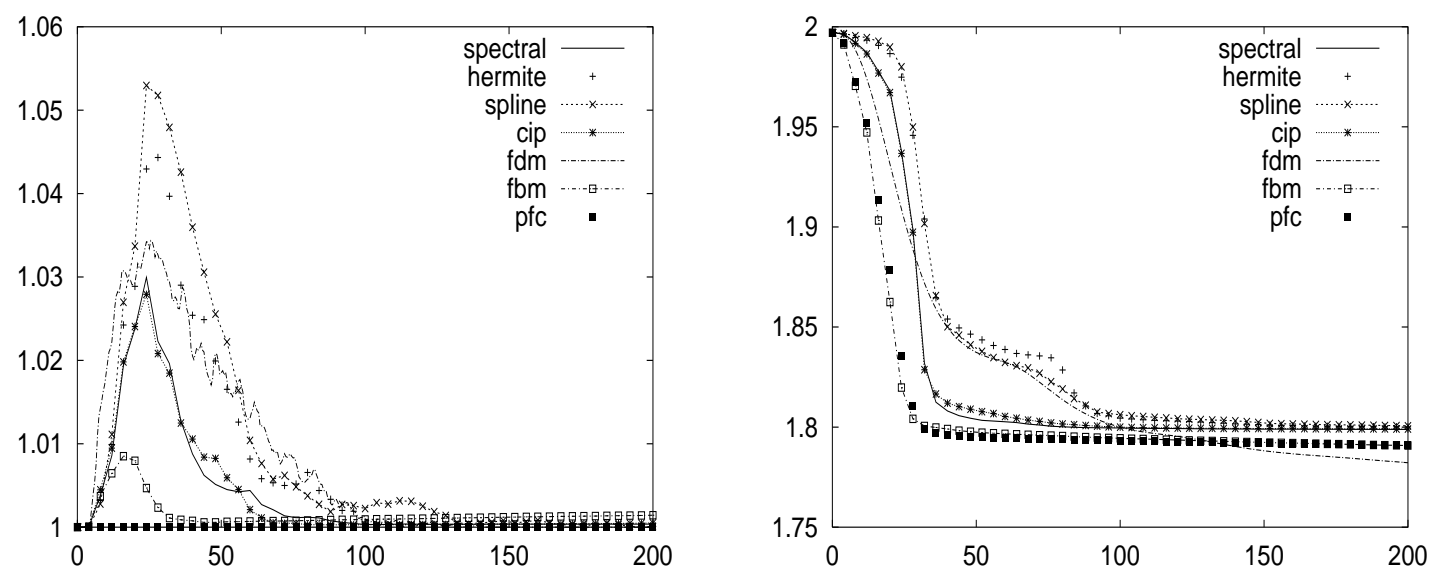

Figure 5: Time development of $L^{1}$ and $L^{2}$ norms of $f(t)$ for the nonlinear Landau damping test.

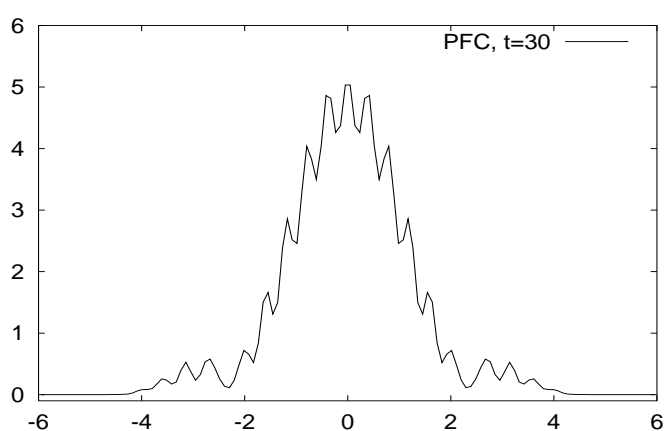

(a)

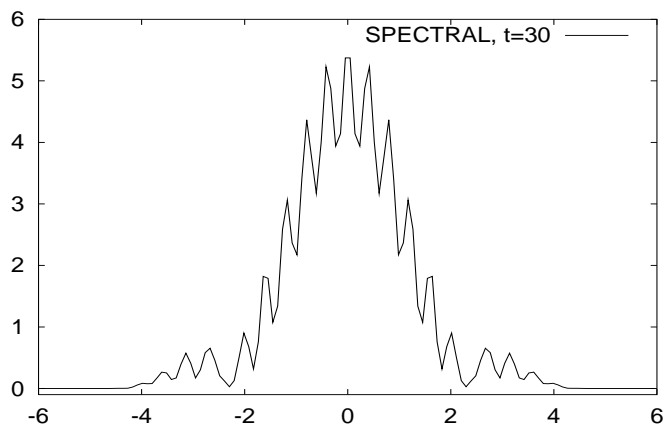

(c)

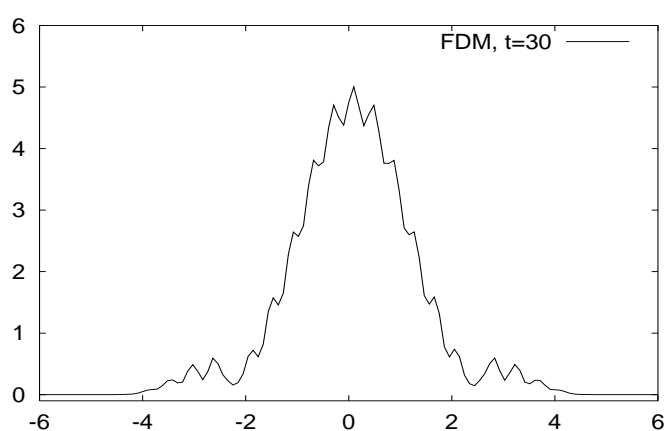

(b)

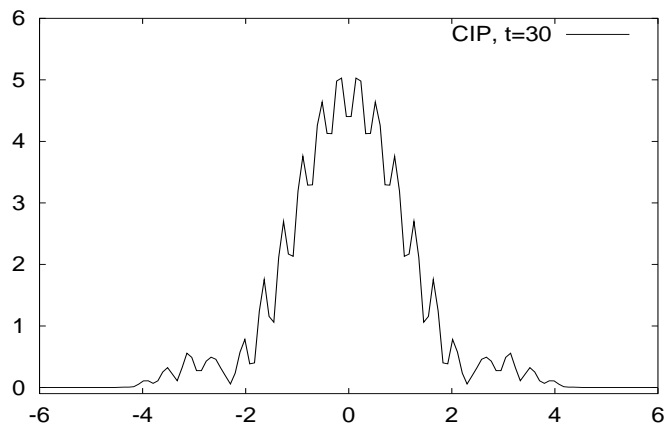

(d)

Figure 6: The space integrated distribution function at time $t=30 \omega_{p}^{-1}$ in space obtained with (a) the PFC scheme, (b) the FDM, (c) the spectral algorithm, (d) the CIP method for the nonlinear Landau damping test. 
with $\alpha=0.01, k=0.5$, and $L=2 \pi / k$. We are using a number of cells $N_{x}=64$ in the $x$-direction, and $N_{v}=64,128$ in the $v$-direction with $v_{\max }=5$, and $\Delta t=1 / 8$ for conservative, spectral and semi-Lagrangian methods which are not restricted by a CFL condition, whereas $\Delta t=1 / 40$ for the Finite Difference Method. From time $t \simeq 20 \omega_{p}^{-1}$ to $t \simeq 40 \omega_{p}^{-1}$, the instability grows rapidly and a hole structure appears. After $t=45 \omega_{p}^{-1}$ until the end of the simulation, trapped particles oscillate in the electric field and the vortex rotates. Fig. 7 shows the time development of the discrete $L^{2}$ norm and of the kinetic entropy for the different methods. For this test nonlinearities are less important than for the previous ones and the decay of the kinetic entropy is smaller. The variations of $H(t)=-\sum f_{i}(t) \ln \left(f_{i}(t)\right)$ for FDM and semi-Lagrangian methods with a Hermite interpolation are less important than for the other methods, but the $x-v_{x}$ projection, plotted in Fig. 8, shows that strong instabilities hold for such methods. The approximation obtained by the different schemes using $64 \times 64$ points is compared with the approximation obtained using $512 \times 1024$ points. Of course, the grid size is too coarse to detail thin filaments developed by the solution. The Flux Conservative methods seem to give a good approximation of the average of the solution on the mesh, whereas the semi-Lagrangian method with a cubic spline interpolation follows thin details of the solution for longer times. Let us mention that in their paper [16], Nakamura and Yabe have compared the CIP method with the well known PIC method. In this case, Eulerian schemes give a better approximation than particle methods.
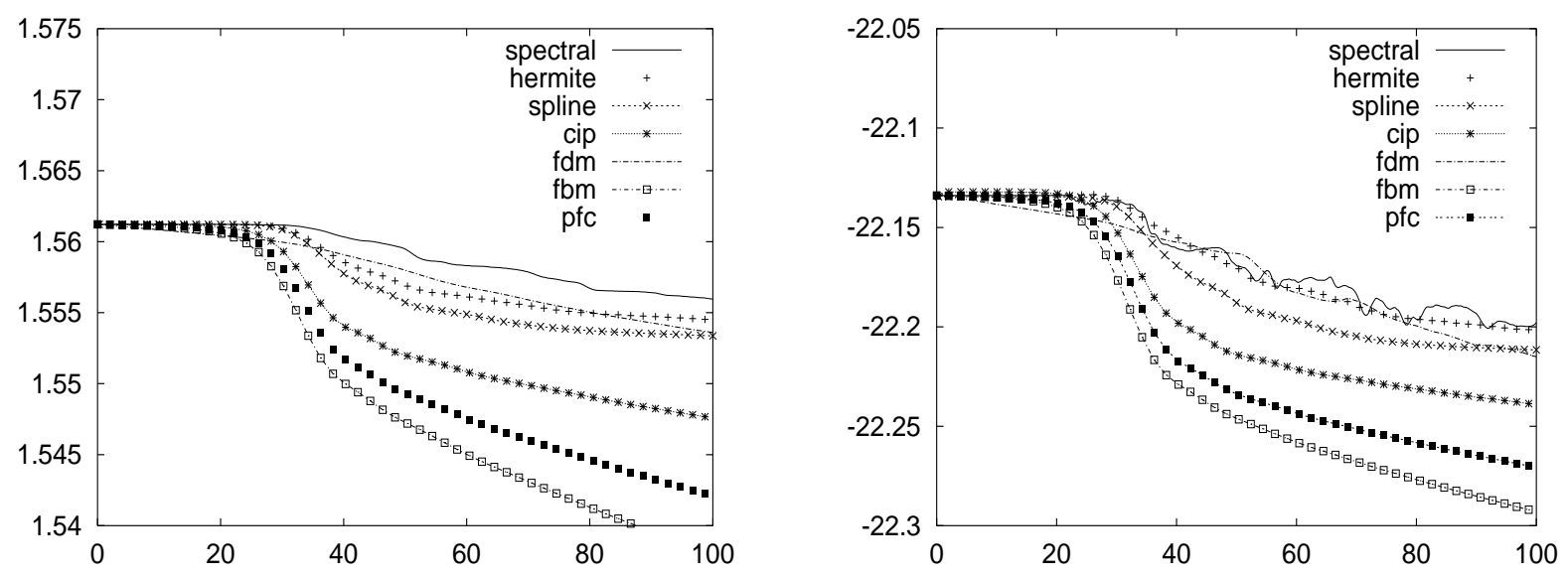

Figure 7: Time development of numerical $L^{2}$ norm and entropy of $f(t)$ for the two stream instability test.

\subsection{The two dimensional Vlasov-Poisson system.}

\subsubsection{The nonlinear Landau damping in 2D.}

The initial condition is set to

$$
f_{0}\left(x, y, v_{x}, v_{y}\right)=\frac{1}{2 \pi} \exp \left(-\left(v_{x}^{2}+v_{y}^{2}\right) / 2\right)\left(1+\alpha\left(\cos \left(k_{x} x\right)+\cos \left(k_{y} y\right)\right)\right),
$$




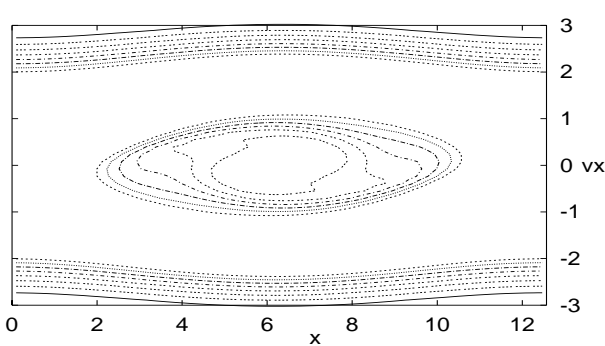

(a)

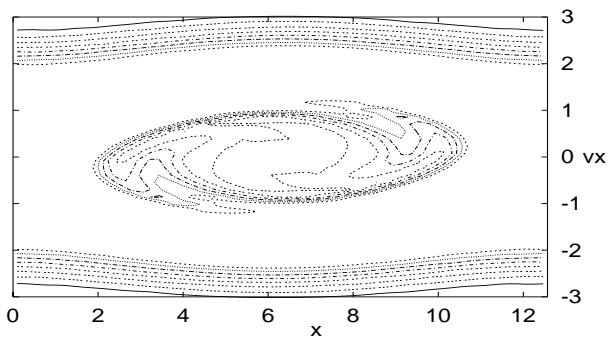

(c)

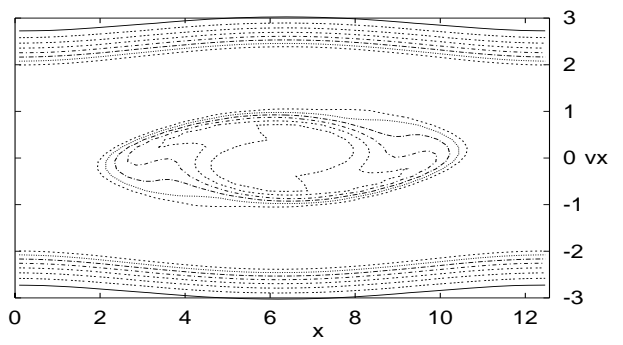

(e)

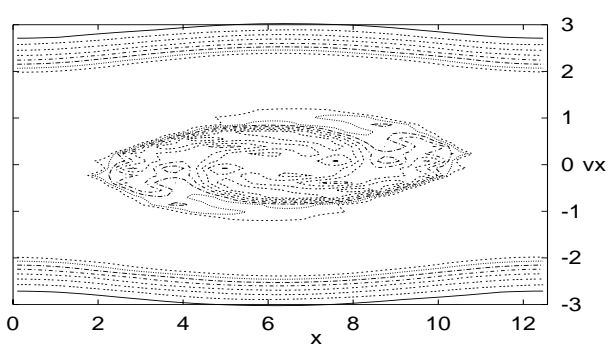

(g)

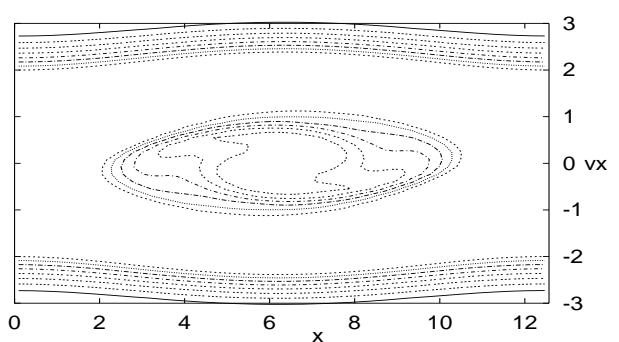

(b)

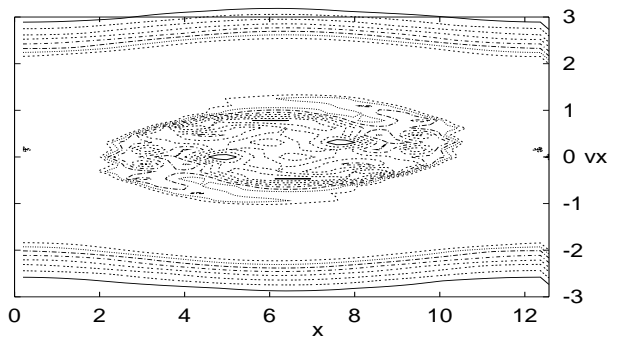

(d)

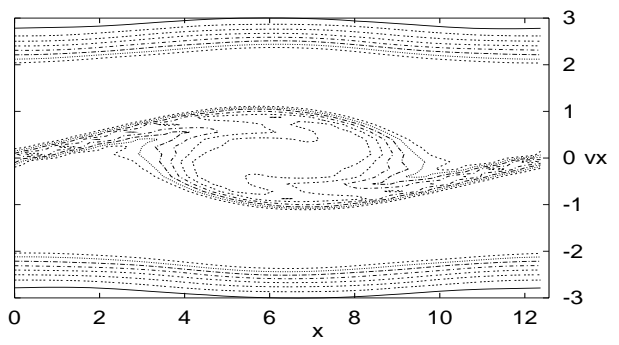

(f)

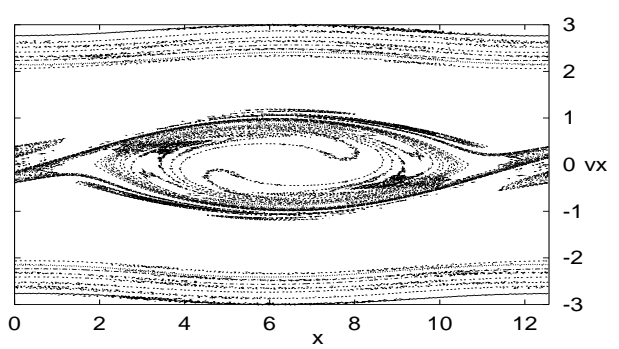

(h)

Figure 8: The $x-v_{x}$ projection of the distribution function $(32 \times 64$ points $)$ at time $t=75 \omega_{p}^{-1}$ obtained with (a) the FBM, (b) the PFC scheme, (c) the spectral algorithm, (d) the FDM, (e) the CIP method, (f) the semi-Lagrangian method with a cubic spline reconstruction, $(g)$ the semiLagrangian method with a Hermite interpolation, (h) an almost "exact" solution $(512 \times 1024$ points) for the two stream instability test. 
with $\alpha=0.5$, the velocity space is truncated at $v_{m a x}=6$, the wave numbers are $k_{x}=k_{y}=0.5$, and the length of the periodic box in the physical space is $L_{x}=L_{y}=4 \pi$. Finally, the four dimensional grid contains 32 points per direction and the time step is set to $\Delta t=1 / 8$. $>$ From the symmetry of the initial data, the evolution of two components of the electric field are identical.

The numerical simulation of nonlinear Landau damping in the four dimensional phase space is a difficult problem since the number of grid points is strongly limited by computer memory, and examples of simulations are not frequent in the literature. Indeed, it is necessary to use a high order scheme, which kills spurious oscillations in order to obtain accurate results. The Fig. 9 shows the evolution of the electrical energy obtained by the PFC scheme and the semi-Lagrangian method using cubic spline interpolation. On the one hand, the local reconstruction highly reduces the computational cost and on the other hand slope correctors avoid to introduce numerical instabilities.
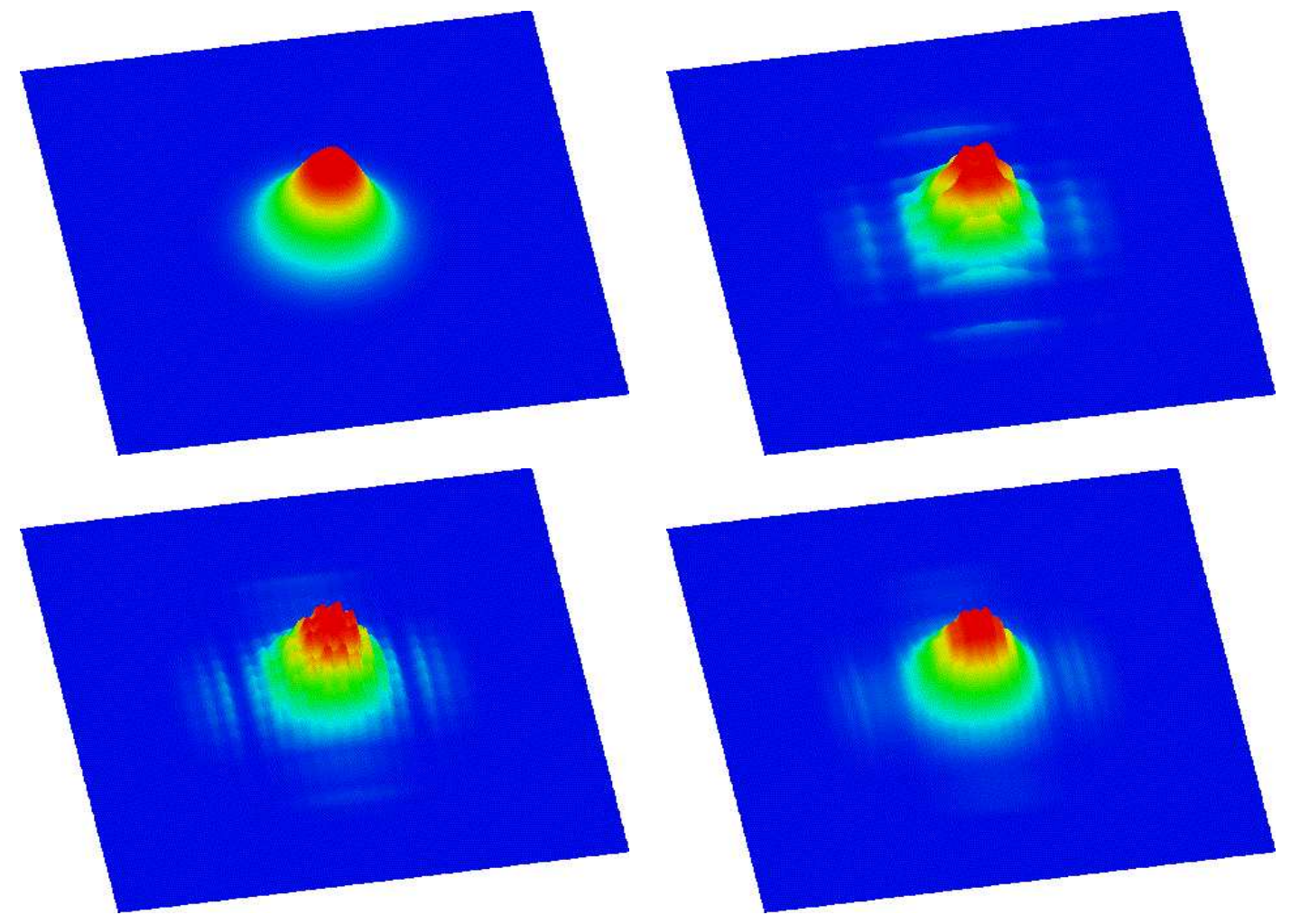

Figure 9: Time development of the $v_{x}-v_{y}$ projection of the distribution function obtained with the PFC scheme $(32 \times 32 \times 128 \times 128)$ for the nonlinear Landau damping $2 D$.

\section{Conclusions.}

At the beginning of numerical simulation of plasmas where computer power was such that only 1D models could be simulated, Particle In Cell methods coexisted with direct Vlasov solvers, and many such solvers can be found in the literature of the 70s. Then in the $80 \mathrm{~s}$ and $90 \mathrm{~s}$ as people could 


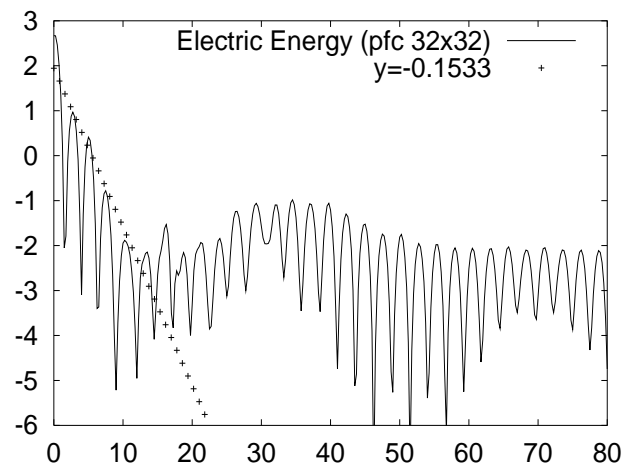

(a)

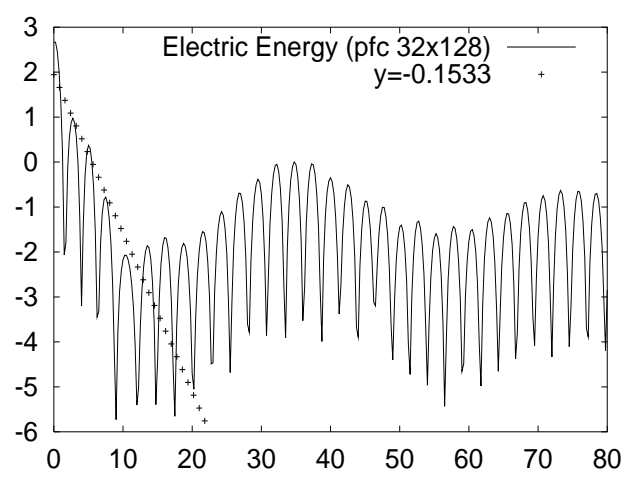

(c)

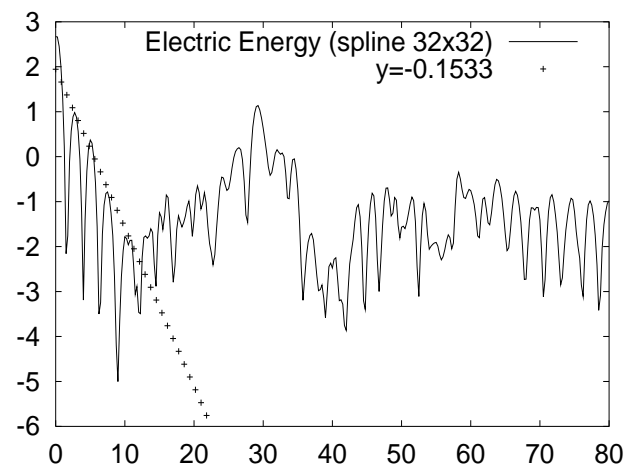

(b)

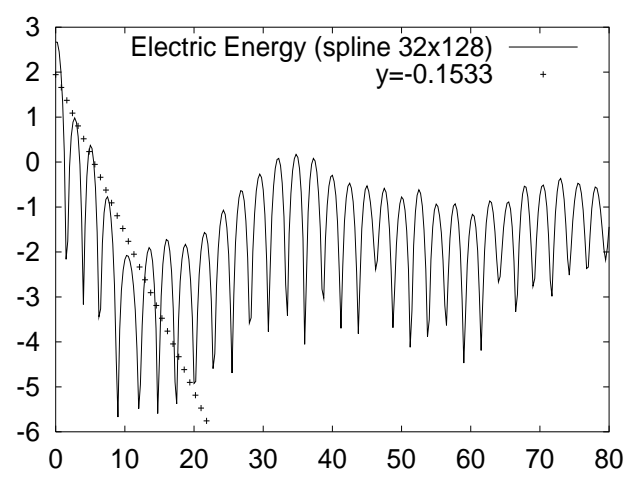

(d)

Figure 10: Time development of the electric energy in logarithm scale obtained with (a) the PFC scheme $(32 \times 32)$, (b) the SL Spline $(32 \times 32)$ (c) PFC scheme $(32 \times 128)$ (d) the SL Spline $(32 \times 128)$ for the nonlinear Landau damping $2 D$.

\begin{tabular}{|c|c|c|}
\hline Number of processors & PFC scheme & SL SPLINE method \\
\hline 2 processors & $9 \mathrm{~h} 03 \mathrm{~min}$ & $12 \mathrm{~h} 42 \mathrm{~min}$ \\
\hline 4 processors & $4 \mathrm{~h} 24 \mathrm{~min}$ & $6 \mathrm{~h} 16 \mathrm{~min}$ \\
\hline 8 processors & $1 \mathrm{~h} 51 \mathrm{~min}$ & $3 \mathrm{~h} 07 \mathrm{~min}$ \\
\hline 16 processors & $0 \mathrm{~h} 59 \mathrm{~min}$ & $1 \mathrm{~h} 41 \mathrm{~min}$ \\
\hline
\end{tabular}

Table 3: Total computation time for PFC and SL PLINE methods with respect to the number of processors for a grid size $32 \times 32 \times 128 \times 128$ points for the nonlinear Landau damping $2 D$. 
perform 2 dimensional and sometimes even 3 dimensional simulation with PIC codes and get useful information out of it direct Vlasov solvers lost their interest for most people. Even though it is a fact that Monte Carlo methods and in particular PIC simulations become numerically the more interesting as the dimension increases, there becomes room again for Eulerian Vlasov solvers as very powerful, as well in CPU speed as in memory size, parallel computers are now available. It is easy nowadays to perform a realistic $2 \mathrm{D}$ kinetic plasma simulation with a direct Vlasov solver and some toy $3 \mathrm{D}$ simulations have also been performed. The numerics on which such methods are based are very different from those of PIC simulations. This fact alone, makes it interesting to have such a solver in one's simulation tool-box in order to benchmark once and again a PIC code against it. And for some specific problems needing high accuracy and low noise they should be the preferred method. As we mentioned, statistical noise is absent from direct Vlasov computations, but they have other flaws which we presented and analyzed here. Among the methods we presented there is no clear winner, each method having its pros and cons. And depending on the problem being solved, one might want to use one or another method. We hope that the information given in this paper will help making this choice.

\section{References}

[1] A. Arakawa, Computational Design for Long-Term Numerical Integration of the Equation of Fluid Motion: Two dimensional Incompressible Flow. Part 1. J. Comput. Phys., 1 (1) (1966) pp. 119-143. Reprinted in J. Comput. Phys., 135 (1997) pp. 103-114.

[2] J. P. Boris, D. L. Book, Solution of continuity equations by the Method of Flux-Corrected Transport. J. Comput. Phys., 20: (1976) pp. 397-431.

[3] F. Bouchut, F. Golse, M. Pulvirenti, Kinetic equations and asymptotic theory, GauthierVillars, Series in applied mathematics, (2000)

[4] Cheng, G. Knorr J. Comput. Phys., 22: (1976) pp. 330-348.

[5] A. J. Klimas, A method for overcoming the velocity space filamentation problem in collisionless plasma model solutions. J. Comput. Phys., 68: (1987) pp. 202-226.

[6] A. Klimas, W. M. Farrell, A Splitting Algorithm for Vlasov Simulation with Filamentation Filtration. J. Comput. Phys., 110: (1994) pp. 150-163.

[7] M. R. Feix, P. Bertrand, A. Ghizzo, Eulerian codes for the Vlasov equation Series on Advances in Mathematics for Applied Sciences 22, Kinetic Theory and Computing (1994) pp. 45

[8] E. Fijalkow, A numerical solution to the Vlasov equation. Comput. Phys. Communications, 116: (1999) pp. 319-328.

[9] F. Filbet, E. Sonnendrücker, Conservative Numerical schemes for the Vlasov equation to appear in J. Comput. Phys. 
[10] A. Ghizzo, P. Bertrand, M. Shoucri, T. W. Johnston, E. Filjakow, M. R. Feix, A Vlasov code for the numerical simulation of stimulated Raman scattering J. Comput. Phys. 90 (1990) pp 431.

[11] G. Manfredi, Long time behavior of non linear Landau damping. Phys. Rev. Letters., 79 -15: (1997) pp. 2815-2818.

[12] R. Robert and J. Sommeria, Statistical equilibrium states for two-dimensional flows J. Fluid. Mech. 229 (1991) pp. 291-310.

[13] M. Shoucri, G. Knorr, Numerical integration of the Vlasov equation. J. Comput. Phys., 14 -1: (1974) pp. 84-92.

[14] E. Sonnendrücker, J. Roche, P. Bertrand, A. Ghizzo, The Semi-Lagrangian Method for the Numerical Resolution of Vlasov Equations. J. Comput. Phys. , 149: (1998) pp.201-220.

[15] E. Sonnendrücker, J.J. Barnard, A. Friedman, D.P. Grote, S.M. Lund, Simulation of heavy ion beams with a semi-Lagrangian Vlasov solver, Nuclear Instruments and Methods in Physics Research, Section A, 464, no. 1-3, (2001) pp. 653-661.

[16] Takashi Nakamura, Takashi Yabe, Cubic interpolated propagation scheme for solving the hyper-dimensional Vlasov-Poisson equation in phase space. Comput. Phys. Communications, 120: (1999) pp. 122-154. 